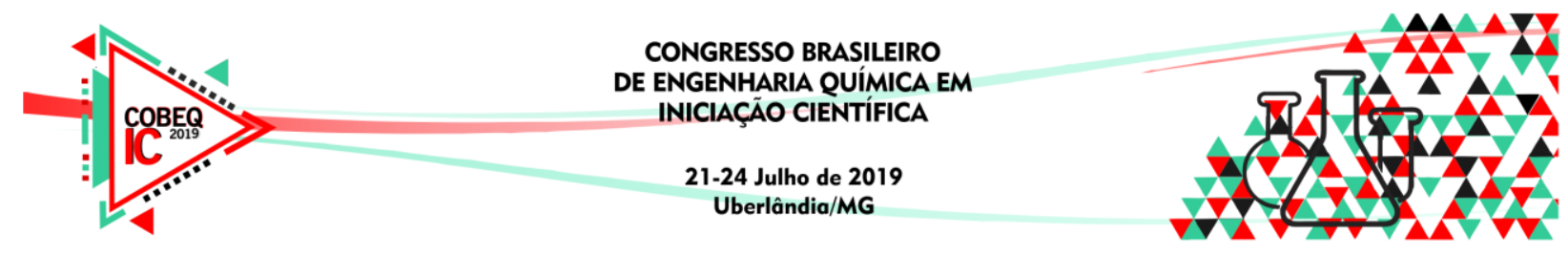

\title{
AVALIAÇÃO DO ENDOCARPO DO FRUTO DA Terminalia catappa Linn COMO ADSORVENTE NA ADSORÇÃO DE CORANTES
}

\author{
G. M. CHEMP ${ }^{1}$, L. V. A. JURADO ${ }^{1}$ e M. F. MENDES ${ }^{1}$ \\ ${ }^{1}$ Universidade Federal Rural do Rio de Janeiro, Departamento de Engenharia Química \\ Laboratório de Termodinâmica Aplicada e Biocombustíveis \\ E-mail para contato: marisamf@ufrrj.br
}

\begin{abstract}
RESUMO - Com o crescimento constante dos polos industriais em grande parte do mundo, ocorre simultaneamente o aumento da poluição dos recursos hídricos. Uma das substâncias poluentes mais nocivas é o corante, que faz parte dos efluentes oriundos de diversos segmentos da indústria, como a têxtil. Entre os métodos disponíveis para a remoção de corantes da água, a adsorção tem se destacado como uma técnica atrativa, devido às suas vantagens como reutilização de biomateriais, baixo custo operacional, tempo de operação curto e alta eficiência. Nesse contexto, o presente estudo propõe avaliar o potencial de adsorção do endocarpo do fruto da árvore Terminalia Catappa Linn no processo de remoção de corantes orgânicos. Os dados experimentais foram medidos, em triplicata, fixando a massa de adsorvente $(0,150 \mathrm{~g})$, granulometria (80 a 270 mesh e 45 a 80 mesh), velocidade de agitação (180 rpm), tempo de contato (60 minutos) e concentração de corante de $104 \mathrm{ppm}$. Foi realizada uma análise do estudo de cinética testando as granulometrias diferentes, com o intuito de analisar o poder de adsorção. No final do processo, foi comprovado que as duas granulometrias estudadas apresentaram um bom potencial de remoção dos corantes e que o modelo cinético de pseudo-segunda ordem correlacionou melhor os dados experimentais.
\end{abstract}

\section{INTRODUÇÃO}

Nos últimos anos, tem-se observado o aumento da contaminação química da água, causada por diferentes poluentes orgânicos e inorgânicos. As indústrias têxteis, por meio dos processos de tinturaria e lavagem, geram um grande volume de efluentes contaminados com corantes, como o alaranjado de metila, que são utilizados nos processos de produção. Os corantes são compostos de difícil degradação e altamente tóxicos para o meio ambiente e, devido a isso, observou-se a necessidade de desenvolver tratamentos adequados para a remoção dessas substâncias dos corpos hídricos (Nascimento et al., 2014).

Os resultados de alguns métodos físico-químicos para o tratamento desses efluentes como a eletroquímica, precipitação, filtração, ozonização, entre outros, mostram que esses procedimentos não têm sido eficazes. Isso ocorre devido ao fato de que os corantes possuem em sua estrutura química anéis aromáticos, grupos aminas, grupos sulfônicos e íons metálicos que, em consequência disso, são de difícil degradação (Rutz et al., 2007). 


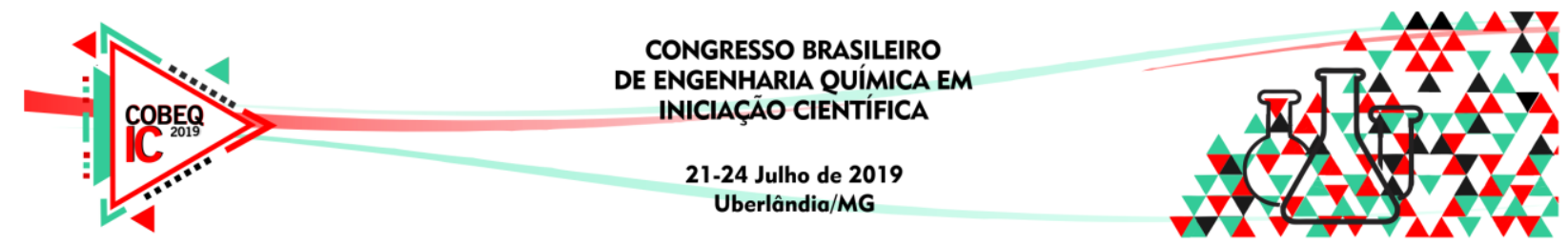

Assim, notou-se a importância de utilizar novas técnicas para tratar efetivamente os efluentes, de modo a diminuir a contaminação. Uma técnica bastante estudada é o processo de adsorção. Um dos adsorventes mais utilizados comercialmente para remover os corantes dos efluentes industriais é o carvão ativado, pois ele possui área superficial alta e muitos poros na sua estrutura. No entanto, a sua utilização é limitada pelo seu custo elevado; em vista disso, existe uma crescente necessidade pela busca de novos materiais adsorventes com baixo custo (Oliveira et al., 2013).

A substituição do carvão ativado por adsorventes oriundos de biomassas residuais tem se mostrado promissora, visto que o número de pesquisas de remoção de corantes, metais pesados, fósforo, nitratos, dentre outros contaminantes de águas e efluentes têm exposto resultados relevantes, ainda mais quando há uso da biomassa "in natura" e o uso de biomassas com modificações superficiais e funcionalização (Hafshejani et al., 2016).

Dentre as possibilidades, encontrou-se a Terminalia Catappa Linn, que é uma árvore grande, com casca cinza lisa, endocarpo duro que envolve as sementes comestíveis e os ramos espiralados. O fruto dessa árvore, comumente chamado de "amêndoa indiana", é composto por caroço $(10,32 \%)$, cobertura fibrosa $(8,97 \%)$, casca $(34,08 \%)$ e endocarpo duro $(46,63 \%)$. Além do ciclo biológico natural, uma quantidade significativa de casca da fruta é descartada como uma biomassa agrícola residual, já que essa árvore tem muitas aplicações de madeira. No presente estudo, parte do fruto foi escolhido como o precursor para a preparação de adsorventes de corantes (Inbaraj et al., 2006).

Frente a isso, o objetivo desse estudo é analisar o desempenho do endocarpo do fruto da Terminalia catappa Linn, no processo de adsorção do corante alaranjado de metila em soluções aquosas, utilizando diferentes granulometrias para comparar e verificar em qual ocorre o processo de adsorção de modo mais efetivo.

\section{MATERIAIS E MÉTODOS}

\subsection{Materiais}

Para os experimentos de adsorção, foi utilizado o seguinte adsorvente: endocarpo do fruto da árvore Terminalia catappa Linn que foi identificado pelo Herbário do Departamento de Botânica da Universidade Federal Rural do Rio de Janeiro (UFRRJ), com registro RBR 44962.

Inicialmente, foram coletadas amostras do fruto nas árvores da Universidade Federal Rural do Rio de Janeiro. Para o preparo da solução sintética foi utilizado o corante alaranjado de metila com concentração de 104 ppm.

\subsection{Métodos}

Tratamento do material adsorvente: As frutas foram lavadas e descascadas. Posteriormente, o material foi seco a $100{ }^{\circ} \mathrm{C}$ numa estufa (DeLeo, tipo A35E) durante $15 \mathrm{~h}$, para a eliminação da umidade. Após a etapa de secagem, o material foi triturado num moinho 


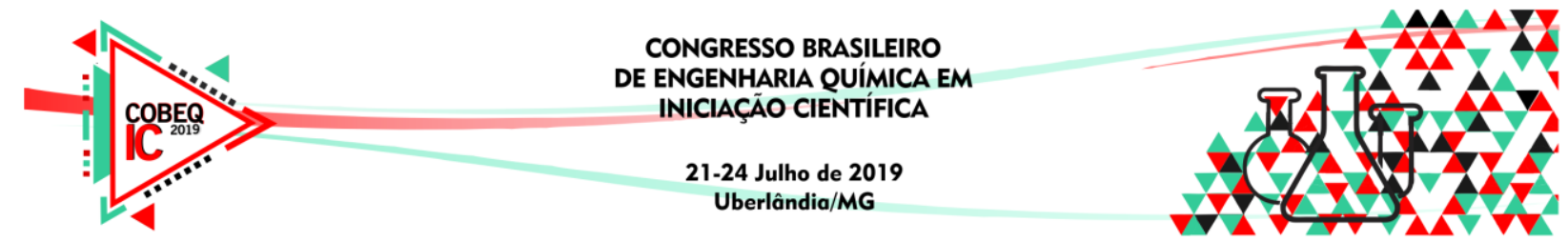

de facas e peneirado para comparar-se as duas faixas granulométricas, atribuindo A1 para a primeira e A2 para a segunda, sendo elas 45 a 80 mesh e 80 a 270 mesh, respectivamente.

Metodologia experimental: Os experimentos cinéticos foram realizados em triplicata, sob agitação de $60 \mathrm{rpm}$, com massa de adsorvente fixa de $0,150 \mathrm{~g}$ em contato com $25 \mathrm{mg} \mathrm{L}^{-1}$ de solução de corante à concentração de $100 \mathrm{mg} \mathrm{L}^{-1}$. Foram retiradas alíquotas nos períodos de $1,3,5,7,10,20,30,50$ e 60 minutos. Os experimentos foram realizados à temperatura de $25^{\circ} \mathrm{C}$. Ao término de cada intervalo, as misturas foram filtradas e coletadas para análise da concentração de corante adsorvida, sendo analisada através de espectrofotômetro UV/visível no comprimento de $465 \mathrm{~nm}$. Os experimentos cinéticos indicaram o tempo necessário para alcançar o equilíbrio de adsorção nas condições experimentais empregadas.

Para os cálculos da quantidade de corante adsorvida no tempo t, qt e no equilíbrio (qe) foi utilizada a equação do balanço de massa, representada pela Equação 1:

$$
q_{t}=\left(C_{0}-C_{t}\right) \frac{V}{m}
$$

sendo $\mathrm{C}_{0}$ e $\mathrm{C}_{\mathrm{t}}$ as concentrações inicial e final de corante $\left(\mathrm{mg} \mathrm{L}^{-1}\right)$, respectivamente, $\mathrm{V}$ o volume da solução de corante (L) e m a massa do adsorvente (g). Quando t é igual ao tempo de contato do equilíbrio, $\mathrm{C}_{\mathrm{t}}=\mathrm{C}_{\mathrm{e}}, \mathrm{q}_{\mathrm{t}}=\mathrm{q}_{\mathrm{e}}$, então a quantidade de corante adsorvido no equilíbrio, qe, é calculada usando a Equação 1.

Modelagem cinética: Com o intuito de investigar o mecanismo de adsorção e as etapas controladoras do processo, modelos cinéticos têm sido desenvolvidos para analisar os dados experimentais (Febrianto et al., 2009). A cinética de adsorção descreve a velocidade com a qual as moléculas do adsorvato são adsorvidas pelo adsorvente. Esta velocidade depende das características físico-químicas do adsorvato (natureza do adsorvato, peso molecular, solubilidade, entre outros), do adsorvente (natureza, estrutura de poros) e da solução ( $\mathrm{pH}$, temperatura, concentração) (Claudino, 2003).

Para investigar o mecanismo do processo de adsorção, os modelos de adsorção de pseudo-primeira ordem (Ho e McKay, 1999) e pseudo-segunda ordem (Chiou e Li, 2003) foram usados para avaliar os dados da taxa de adsorção. O modelo de taxa de pseudo-primeira ordem é expresso na Equação 2:

$$
\ln \left(q_{e}-q_{t}\right)=\ln q_{e}-K_{1} t
$$

sendo $\mathrm{q}_{\mathrm{e}}$ e $\mathrm{q}_{\mathrm{t}}$ as quantidades de corante adsorvido no adsorvente $\left(\mathrm{mg} \mathrm{g}^{-1}\right)$ no equilíbrio e no tempo $\mathrm{t}$, respectivamente, e $\mathrm{k}_{1}$ a constante de taxa $(\mathrm{min})$. $\mathrm{O}$ modelo de taxa de pseudosegunda ordem é representado pela Equação 3:

$$
\frac{t}{q_{t}}=\frac{1}{K_{2} q_{e}{ }^{2}}+\frac{1}{q_{e}} t
$$

sendo $\mathrm{K}_{2}$ a constante da taxa de pseudo-segunda ordem $\left(\mathrm{g} \mathrm{mg}^{-1} \mathrm{~min}^{-1}\right)$. 


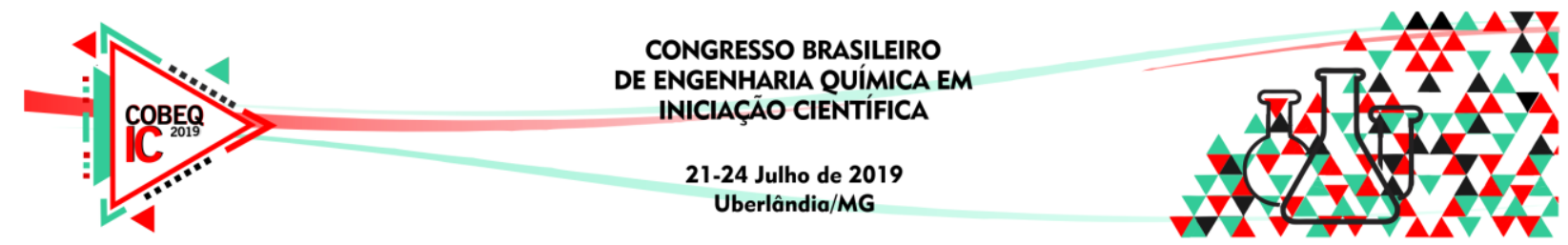

\section{RESULTADOS E DISCUSSÃO}

\subsection{Cinética de Adsorção}

A Figura 1 apresenta os dados experimentais dos ensaios de cinética, os quais foram realizados com a solução sintética do corante alaranjado de metila, com o adsorvente em duas diferentes faixas granulométricas. Esse estudo permitiu obter o tempo de equilíbrio para se atingir a capacidade máxima de adsorção dos adsorventes A1 e A2, assim como a cinética do processo.

Figura 1 - Cinética da adsorção do corante alaranjado de metila, usando as amostras de adsorvente $\mathrm{A} 1$ e $\mathrm{A} 2$ a $25^{\circ} \mathrm{C}$.

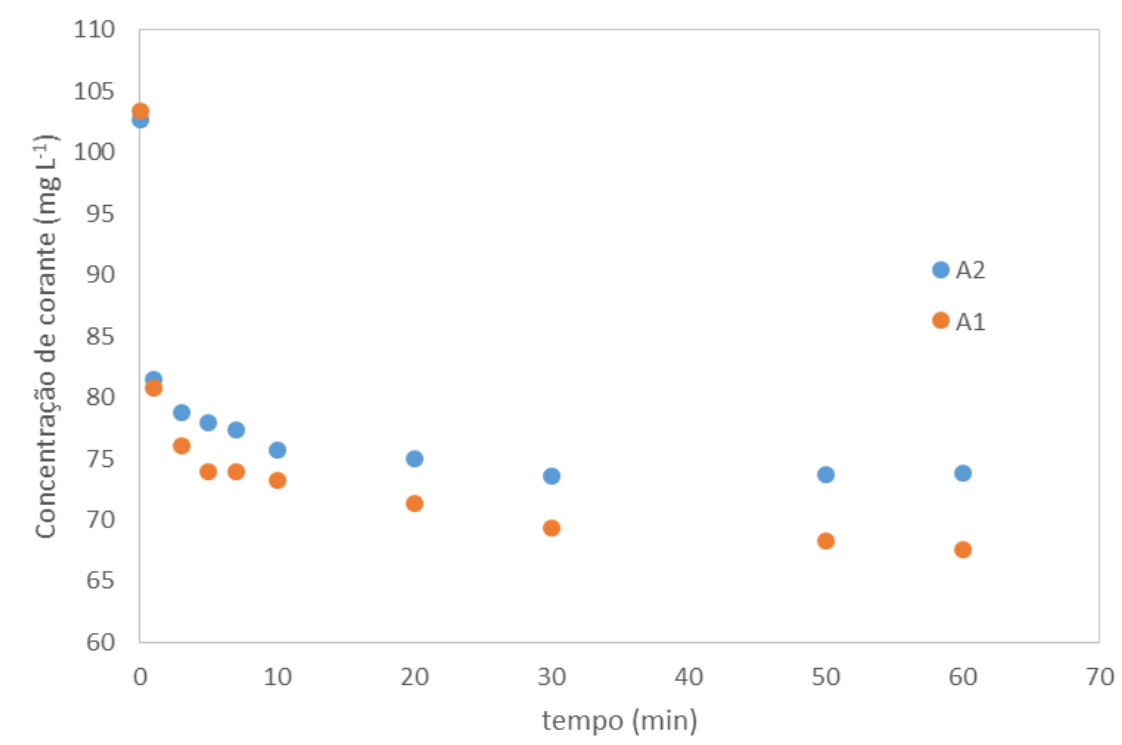

A partir do gráfico, é possível visualizar que o tempo necessário para os sistemas entrarem em equilíbrio é inferior a 60 minutos para as duas granulometrias estudadas e que a remoção de corante diminuiu com o tempo de contato até o equilíbrio. O adsorvente $\mathrm{A} 2$, apresentou uma remoção de $28,38 \%$ e o adsorvente A1 apresentou uma remoção máxima de $34,64 \%$. Os percentuais obtidos podem ser comparados com o do Neto et al. (2015), em que foi estudado o poder de adsorção da Argila Bentonita-Chocolate frente ao alaranjado de metila e obteve-se uma remoção de $14 \%$ em 60 minutos. Com isso, nota-se uma maior eficiência do adsorvente utilizado no presente trabalho.

Através dos dados experimentais obtidos no estudo cinético e com a finalidade de conhecer algumas características sobre a cinética de adsorção do corante, foram aplicados dois modelos cinéticos: pseudo-primeira ordem e pseudo-segunda ordem. Os dados experimentais, com as duas amostras, foram modelados linearizando as equações e comparando-se os coeficientes de correlação. Os gráficos de $\ln (\mathrm{qe}-\mathrm{qt})$ em função de $\mathrm{t}$ para o modelo de pseudoprimeira ordem e t/qt em função de t para o modelo de pseudo-segunda ordem, foram construídos e são apresentados na figura 2, e os parâmetros das regressões lineares empregando os modelos de pseudo-primeira e pseudo-segunda ordem estão apresentados na Tabela 1. 


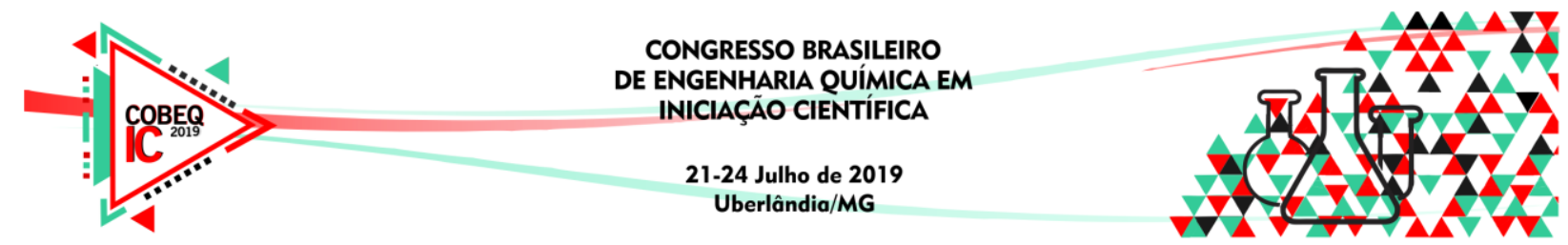

Figura 2 - Modelos de (a) pseudo-primeira ordem para A1; (b) pseudo-segunda ordem para A1; (c) pseudo-primeira ordem para A2 e (d) pseudo-segunda ordem para A2
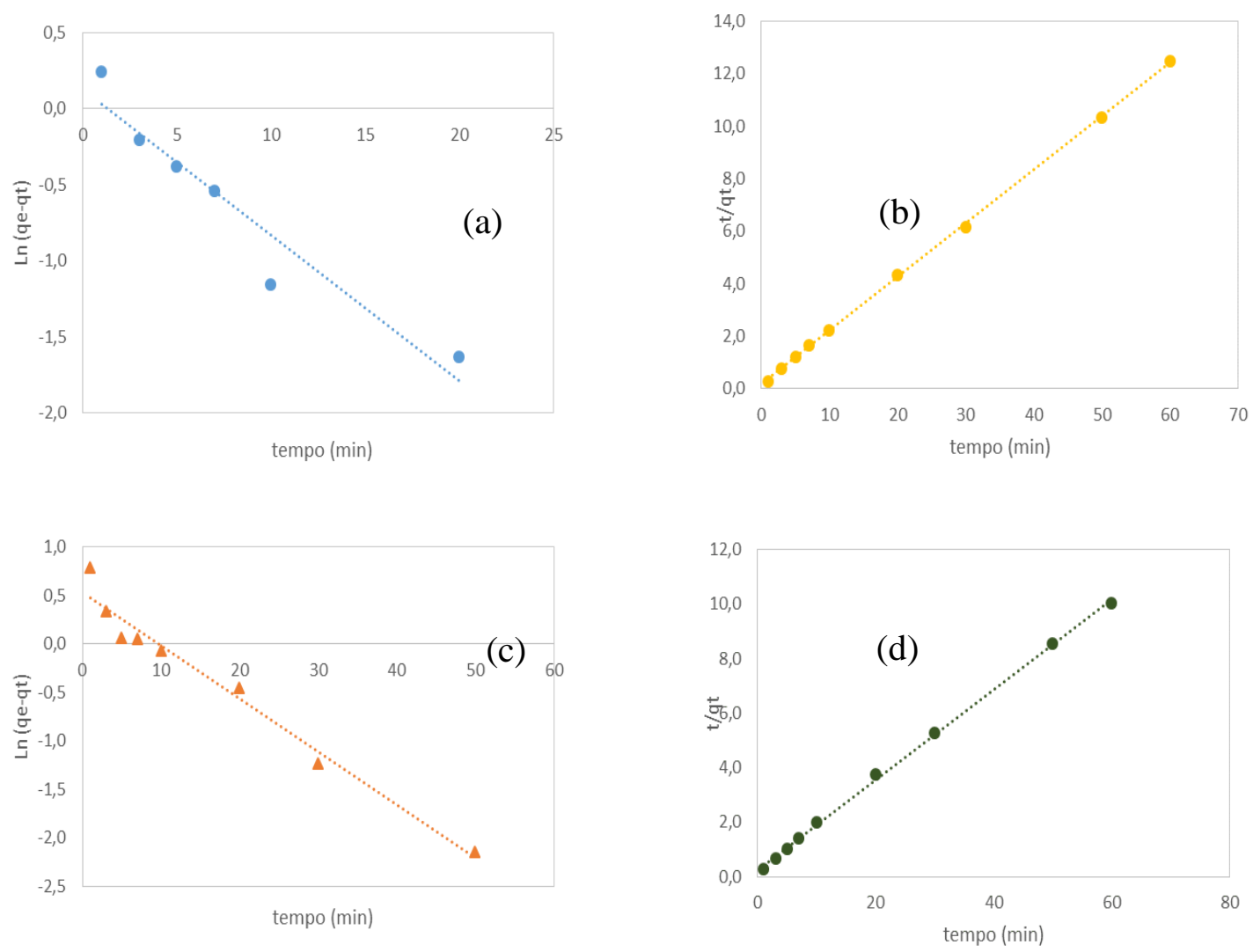

Tabela 1 - Parâmetros de regressão linear para os dois modelos estudados

\begin{tabular}{|c|c|c|c|c|c|c|c|}
\hline \multirow{2}{*}{ Adsorvente } & \multirow{2}{*}{$\mathrm{q}_{\mathrm{e}}^{*}$} & \multicolumn{3}{|c|}{ Pseudo-primeira Ordem } & \multicolumn{3}{c|}{ Pseudo-segunda Ordem } \\
\cline { 3 - 8 } & & $\mathrm{K}_{1}$ & $\mathrm{q}_{\mathrm{e}}$ & $\mathrm{R}_{2}$ & $\mathrm{~K}_{1}$ & $\mathrm{q}_{\mathrm{e}}$ & $\mathrm{R}_{2}$ \\
\hline \hline $\mathrm{A} 1$ & 4,8131 & 0,0955 & 1,1290 & 0,9225 & 0,1136 & 4,8924 & 0,9998 \\
\hline $\mathrm{A} 2$ & 5,9730 & 0,0547 & 1,6967 & 0,9710 & 0,1136 & 6,0423 & 0,9991 \\
\hline
\end{tabular}

$\mathrm{q}_{\mathrm{e}}^{*}$ é a $\mathrm{q}_{\mathrm{e}}$ experimental

Através dos resultados obtidos pela regressão dos dois modelos, é possível observar que ambos apresentaram coeficientes de correlação acima de 0,9 , sendo mais próximo de 1 para o adsorvente A1 $(0,9998)$ e A2 $(0,9991)$ no modelo de pseudo-segunda ordem. Ao comparar o valor de $\mathrm{q}_{\mathrm{e}}$ com o dado obtido experimentalmente, é possível notar que o modelo de pseudosegunda ordem obteve um melhor resultado, pois os valores estão mais próximos de $\mathrm{q}_{\mathrm{e}}^{*}$. Já o modelo de pseudo-primeira ordem distanciou-se do valor experimental. Portanto, é possível inferir que o modelo de pseudo-segunda ordem foi o que melhor ajustou os dados, tanto para $\mathrm{o}$ adsorvente A1 quanto para A2. 


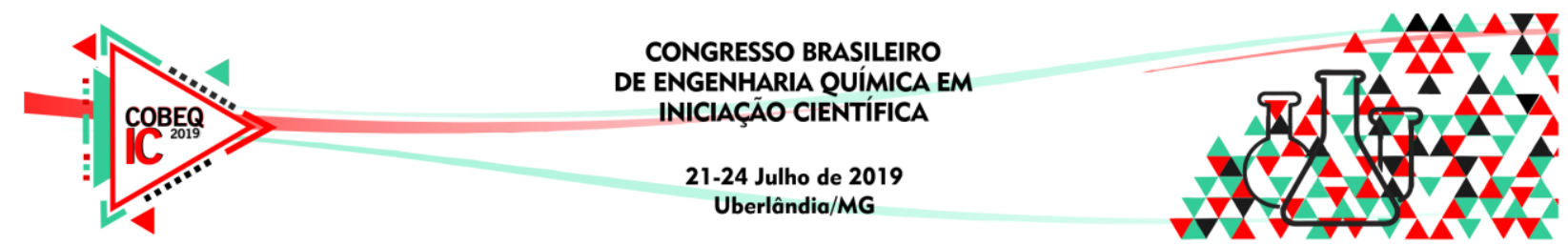

\section{CONCLUSÃO}

O adsorvente estudado dispõe de um bom poder de adsorção. Os resultados apontam que o adsorvente A1 obteve um percentual de remoção um pouco maior, quando comparado ao adsorvente A2, sendo 6,26\% o percentual de diferença da adsorção entre os dois. Visto que a diferença é baixa, a proposta é que seja feita o uso das duas granulometrias juntas, de modo que todo o material seja aproveitado. Para os dois, o tempo de equilíbrio foi entre 30 minutos e o processo de adsorção ocorreu de modo espontâneo. Além disso, os dados apontam que a remoção do corante diminuiu com o aumento do tempo de contato até atingir o equilíbrio. $\mathrm{O}$ modelo cinético que melhor correlacionou os dados experimentais foi o de pseudo-segunda ordem.

\section{REFERÊNCIAS}

CHIOU, M. S.; LI, H. Y. Adsorption behavior of reactive dye in aqueous solution on chemical cross-linked chitosan beads. Chemosphere, v. 50, n. 8, p. 1095-1105, 2003.

CLAUDINO, A., Preparação de carvão ativado a partir de turfa e sua utilização na remoção de poluentes. Florianópolis: Universidade Federal de Santa Catarina, Dissertação (Mestrado), 2003.

FEBRIANTO, J.; KOSASIH, AN.; SUNARSO, J.; JU, Y.H.; INDRASWATI, N.; ISMADJI, S., Equilibrium and kinetic studies in adsorption of heavy metals using biosorbent: a summary of recent studies, Journal of Hazardous Materials. v. 162, p. 616-620, 2009.

HAFSHEJANI, L. D.; HOOSHMAND, A.; NASERI, A. A.; MOHAMMADI, A. S.; ABBASI F.; BHATNAGAR, A. Removal of nitrate from aqueous solution by modified sugarcane bagasse biochar. Ecological Engineering, v. 95, p.101-111, 2016.

HO, Y. S.; McKAY, G. The sorption of lead (II) ions on peat. Wat. Res. v. 33, n. 2, p. 578$584,1999$.

INBARAJ B. S.; SULOCHANA, N. Mercury adsorption on a carbono sorbent derived from fruit shell of Terminalia catappa, Department of Chemistry, India, 2006.

NASCIMENTO, R. F.; LIMA, A. C. A.; VIDAL, C. B.; MELO, D. Q.; RAULINO, G. S. C. Adsorção, aspectos teóricos e aplicações ambientais. Fortaleza: Imprensa Universitária, 2014.

NETO, J. F. D. Processo de Adsorção dos Corantes Alaranjado de Metila e RodaminaB por Argilas Esmectíticas da Paraíba, in natura e modificadas. Campina Grande: Universidade Federal de Campina Grande, Dissertação (Mestrado), 2015.

OLIVEIRA, S. P.; SILVA, W. L. L.; VIANA, R. R. Evaluation of the capacity of adsorption of methylene blue die in aqueous solutions in natural kaolinite and intercalated with potassium acetate., Cerâmica 59, p. 338-339, 2013. 